\title{
Fluorine atom induced decreases to the contribution of infrared vibrations to the static dielectric constant of $\mathrm{Si}-\mathrm{O}-\mathrm{F}$ alloy films
}

\author{
G. Lucovskya) and H. Yang \\ Departments of Physics, Chemistry, Materials Sciences and Engineering, and Electrical and Computer \\ Engineering, North Carolina State University, Raleigh, North Carolina 27965-8202
}

(Received 18 February 1997; accepted 31 March 1997)

\begin{abstract}
$\mathrm{Si}-\mathrm{O}-\mathrm{F}$ alloy films deposited by chemical vapor deposition have static dielectric constants, $\boldsymbol{\epsilon}_{s}$, significantly reduced with respect to those of similarly prepared $\mathrm{SiO}_{2}, \sim 3.2$ to 3.4 as compared to 4.0 to 4.2. Infrared absorption spectra provide a basis modeling the molecular structure of these alloys, as well as helping to identify microscopic mechanisms responsible for static dielectric constant reductions. Contributions of electronic and vibrational transitions to $\epsilon_{s}$ are discussed in terms of an empirical chemical bonding model. Ab initio calculations are then used to identify inductive effects of $\mathrm{Si}-\mathrm{F}$ bonds on the properties of $\mathrm{Si}-\mathrm{O}-\mathrm{Si}$ groups that are back-bonded to the $\mathrm{Si}$ atom of that $\mathrm{Si}-\mathrm{F}$ group. The $a b$ initio calculations provide a theoretical framework for understanding why relatively low concentrations of $\mathrm{F}$ atoms; $\sim 10-12$ at. \%, produce significant decreases in $\epsilon_{s}, \sim 22 \%$, as reported for the Si-O-F alloys. (C) 1997 American Vacuum Society. [S0734-2101(97)12203-5]
\end{abstract}

\section{INTRODUCTION}

There is considerable interest in insulating films with static dielectric constants, $\epsilon_{s}$, lower than $\mathrm{SiO}_{2}$ for use as dielectric layers between thin film metal interconnects in integrated circuits. One alloy system that has recently attracted considerable attention for this application is $\mathrm{Si}-\mathrm{O}-\mathrm{F}$. This article

(i) reviews previously published experimental results for the infrared, (IR), optical properties of $\mathrm{Si}-\mathrm{O}-\mathrm{F}$ alloys, ${ }^{1,2}$

(ii) presents new IR data, which when combined with IR data of Refs. 1 and 2, provides the basis for a description of the local bonding arrangements of the incorporated $\mathrm{F}$ alloy atoms,

(iii) characterizes the alloy compositions in a pseudobinary notations,

(iv) explains the major contributing factors to the reductions in $\epsilon_{s}$ atoms by three effects:

(a) replacement of strongly IR-active $\mathrm{Si}-\mathrm{O}-\mathrm{Si}$ with weaker $\mathrm{Si}-\mathrm{F}$ bonds,

(b) chemical induction effects, ${ }^{3}$ whereby $\mathrm{Si}-\mathrm{F}$ bonds produce changes in frequencies and infrared effective charges of the vibration modes of nearest neighbor $\mathrm{Si}-$ $\mathrm{O}-\mathrm{Si}$ bonding groups reducing the contribution of these modes to the $\epsilon_{s}$, and

(c) reductions in alloy density. ${ }^{4}$

The reductions in alloy density, $\sim 5 \%$ for films with $10-12$ at. $\% \mathrm{~F}$, can only account for about $20 \%$ of the total decreases in $\epsilon_{s}$ reported for these films.

On the other hand in Ref. 2 it was demonstrated that decreases in the intensities of $\mathrm{Si}-\mathrm{O}-\mathrm{Si}$ vibrations in the IR absorption spectrum account for a significant fraction of the decreases in the static dielectric constant of $\mathrm{Si}-\mathrm{O}-\mathrm{F}$ alloys with respect to $\mathrm{SiO}_{2}$. However, no attempts were made in

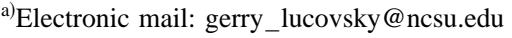

either Refs. 1 or 2 to provide an understanding of why these additional decreases in $\epsilon_{s}$ with $\mathrm{F}$ alloy atom incorporation were so large. For example, a one for one substitution of $\mathrm{Si}-\mathrm{F}$ bonds for $\mathrm{Si}-\mathrm{O}$ bonds is insufficient to explain the large changes that occur. In making this comparison, it is necessary to compare integrated absorption strengths rather than simply peak values of the absorption constants of the $\mathrm{Si}-\mathrm{F}$ and $\mathrm{Si}-\mathrm{O}-\mathrm{Si}$ absorption bands. In this article it is shown that the very large changes in $\epsilon_{s}$ can be understood in terms of additional effects in which the presence of $\mathrm{Si}-\mathrm{F}$ bonding

(i) reduces the dynamic IR effective charge of nearestneighbor IR-active $\mathrm{Si}-\mathrm{O}-\mathrm{Si}$ bond-bending vibrations, and

(ii) increases the frequencies of $\mathrm{Si}-\mathrm{O}-\mathrm{Si}$ bond-stretching vibrations.

Both of these contribute to reductions in $\epsilon_{s}$ as will be demonstrated using an extended Penn Model for the quantifying the contributions of the vibrational modes to $\epsilon_{s} \cdot{ }^{5}$

\section{INFRARED SPECTRA}

The significant changes in IR absorption in $\mathrm{Si}-\mathrm{O}-\mathrm{F}$ alloys with respect to $\mathrm{SiO}_{2}$ have been documented in Refs. 1 and 2 where IR data has been presented in the spectral range from 400 to $4000 \mathrm{~cm}^{-1}$. This range includes the $\mathrm{Si}-\mathrm{O}$ and $\mathrm{Si}-\mathrm{F}$ vibrational modes that make the most significant contributions to $\epsilon_{s}$; i.e., the $\mathrm{Si}-\mathrm{O}-\mathrm{Si}$ asymmetric bondstretching mode which peaks at $\sim 1050-90 \mathrm{~cm}^{-1}$, the silicon monofluoride $(\mathrm{Si}-\mathrm{F})$ bond-stretching mode at $\sim 935 \mathrm{~cm}^{-1}$, the $\mathrm{Si}-\mathrm{O}-\mathrm{Si}$ bond-bending mode at $\sim 810 \mathrm{~cm}^{-1}$, and the $\mathrm{Si}-\mathrm{O}-\mathrm{Si}$ bond-rocking mode $\sim 460 \mathrm{~cm}^{-1}$. Si-F bond-bending modes are expected at frequencies below $400 \mathrm{~cm}^{-1}$; however, our recent IR measurements shown in Fig. 1 indicate that these are not detectable in alloy films with up to about 12 at. \% F. These samples were prepared using conventional direct plasma processing 

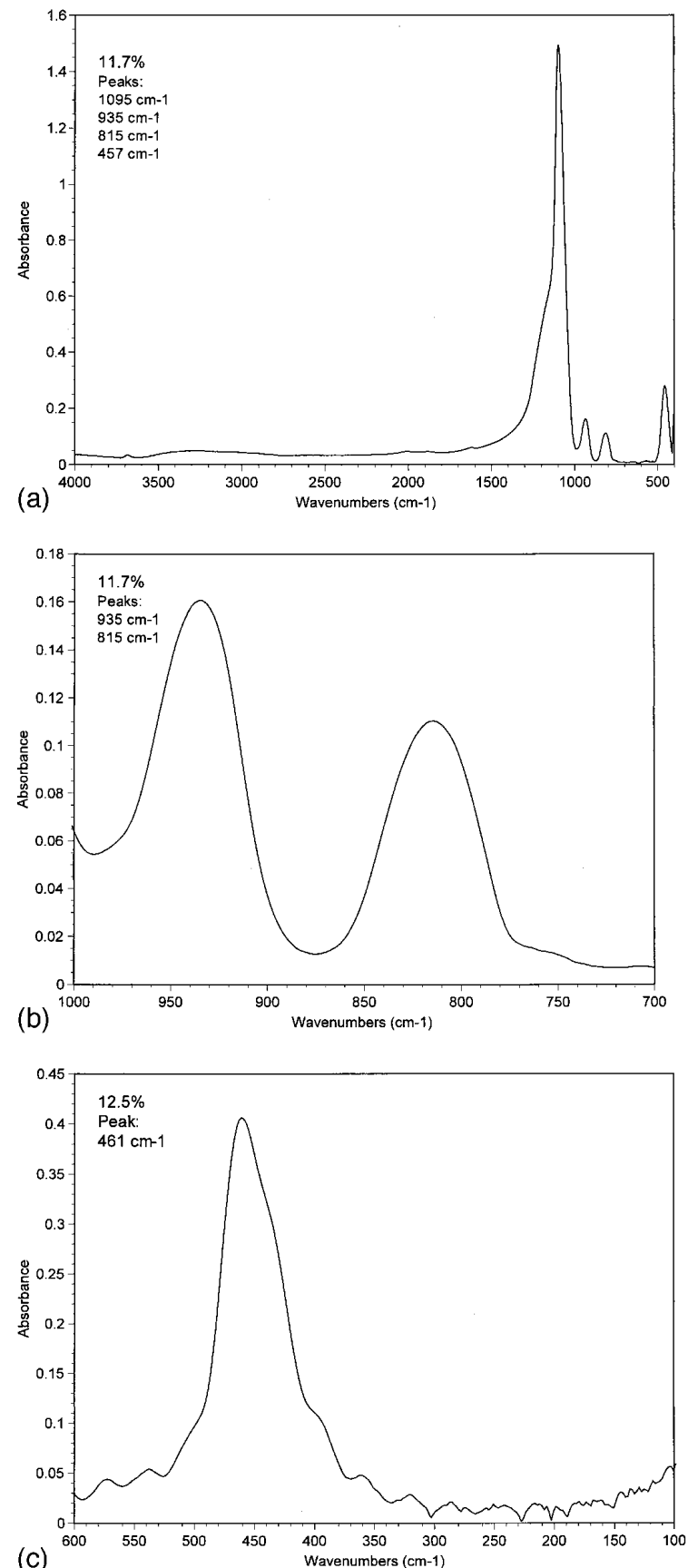

(c)

FIG. 1. IR spectra of a Si-O-F alloys: (a) IR spectra from 400 to $4000 \mathrm{~cm}^{-1}$ for alloy with 11.7 at. \% F; (b) expanded spectrum in the range of the Si-F bond-stretching feature $\left(935 \mathrm{~cm}^{-1}\right)$ and the $\mathrm{Si}-\mathrm{O}-\mathrm{Si}$ bondbending mode $\left(815 \mathrm{~cm}^{-1}\right)$ for alloy with 11.7 at. \% F; and (c) IR spectra from 150 to $400 \mathrm{~cm}^{-1}$ for an $\mathrm{Si}-\mathrm{O}-\mathrm{F}$ alloy with 12.5 at. \% F. The thicknesses of the alloy films with 11.7 and 12.5 at. \% F are essentially the same.

tools, and the $\mathrm{F}$ atom concentrations were determined by $\mathrm{x}$-ray photoelectron spectroscopy. The data in Fig. 1 are for samples of approximately the same thickness. We have presented IR data for alloy films, which may then be compared with IR data for $\mathrm{SiO}_{2}$ films in the corresponding spectral range as presented in Refs. 1 and 2.

Returning first to the data presented in Refs. 1 and 2 in- clude, the most important observations reported for the $\mathrm{Si}-$ $\mathrm{O}-\mathrm{F}$ alloys included the following:

(i) for incorporation of $\mathrm{F}$ to about $10-12$ at. \% there are three significant changes in the IR absorption with respect to $\mathrm{SiO}_{2}$ :

(a) development of a weak $\mathrm{Si}-\mathrm{F}$ bond-stretching absorption at $\sim 935 \mathrm{~cm}^{-1}$,

(b) changes in the $\mathrm{Si}-\mathrm{O}$ bond-stretching band; most notably an increase in the position of the spectral peak with increasing F content, and an accompanying decrease in the half-width, which combine to decrease the integrated IR absorption strength, and

(c) reductions in the integrated IR absorption strengths of the 810 and $465 \mathrm{~cm}^{-1}$ bands with increasing $\mathrm{F}$ content;

(i) reductions in $\epsilon_{s}$ from values of $\sim 4.1-4.2$ to 3.3 \pm 0.1 for $F$ concentrations of $\sim 10-12$ at. $\%$, and

(ii) changes in film properties upon exposure to atmospheric water and low-temperature annealing.

The spectra presented in Fig. 1 show essentially the same changes with respect to $\mathrm{SiO}_{2}$ spectra as noted above in (i) (a) and (b); e.g., compare the spectra in Fig. 1 with those presented in Ref. 1.

It is important to note that there is no definitive spectroscopic evidence for $\mathrm{Si}-\mathrm{F}_{2}$ bonding arrangements in alloys with up to about 12 at. $\% \mathrm{~F}$ in the published literature, ${ }^{1,2,6}$ or in the new IR data presented in this article; e.g., in the form of the scissors and rocking bond-bending modes that are anticipated to occur at a frequencies below about $500 \mathrm{~cm}^{-1}$. Returning to bond-stretching modes, Hayasaka et al. in Ref. 6 have suggested that the development of low and high wavenumber features on the $935 \mathrm{~cm}^{-1} \mathrm{Si}-\mathrm{F}$ absorption are indicative of the symmetric and anti-symmetric bondstretching vibrations of $\mathrm{Si}-\mathrm{F}_{2}$ bonding groups. The splitting between these two features that they attribute to $\mathrm{Si}-\mathrm{F}_{2}$ bonding groups is $\sim 68 \mathrm{~cm}^{-1}$ for $\mathrm{F}$ concentrations of about $6-8$ at. $\%$, and is significantly smaller than what is expected on the basis of kinematic effects for $\mathrm{Si}-\mathrm{F}_{2}$ groups from the atomic masses of $\mathrm{Si}$ and $\mathrm{F}^{7}$ For example, based on the calculations in Ref. 7 a frequency splitting of at least $90 \mathrm{~cm}^{-1}$ is expected for the splitting between the symmetric and antisymmetric $\mathrm{Si}-\mathrm{F}_{2}$ vibrations. Finally, in order to confirm unambiguously the presence of $\mathrm{Si}-\mathrm{F}_{2}$, it is necessary to show the presence of bond-bending scissors and bond-rocking modes. ${ }^{7}$ As noted above, based on the recent IR studies performed in our laboratory which have extended absorption spectra down to $150 \mathrm{~cm}^{-1}$, there is no spectroscopic evidence for either of these types of vibrational modes in samples with up to 12.5 at. \% F (see Fig. 1). In addition, the results shown in Fig. 1 also demonstrate that the two additional spectral features reported for the $\mathrm{Si}-\mathrm{F}$ bond-stretching vibration in Ref. 6 are not intrinsic to $\mathrm{Si}-\mathrm{O}-\mathrm{F}$ alloy films. In particular, comparisons with other studies suggest that the $920 \mathrm{~cm}^{-1}$ feature reported in Ref. 6 is derived from a reaction of the Si-O-F film with water; ${ }^{8}$ i.e., the $920 \mathrm{~cm}^{-1}$ fea- 
local bonding groups in pseudo-binary alloy system

$\left(\mathrm{SiO}_{2}\right)_{x}\left(\mathrm{Si}_{2} \mathrm{O}_{3} \mathrm{~F}_{2}\right) 1-\mathrm{x}$

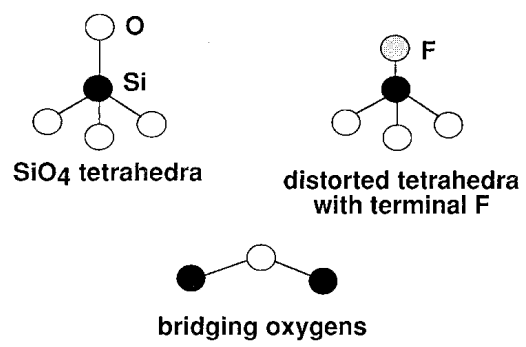

FIG. 2. Local molecular structures in Si-O-F alloys.

ture is due to a displacement of hydrogen bonded $\mathrm{OH}$ groups with respect to $\mathrm{Si}$ atoms. The results of Ref. 6 showed a distinct feature developed at $\sim 920 \mathrm{~cm}^{-1}$, whereas the presence of a feature at about $980 \mathrm{~cm}^{-1}$ was inferred from an attempt to fit the spectral data with three gaussian-shaped bands. Based on the data in Fig. 1, the existence of a discreet mode at $980 \mathrm{~cm}^{-1}$ that is intrinsic to $\mathrm{Si}-\mathrm{O}-\mathrm{F}$ alloys is highly questionable. A similar conclusion can also be drawn from the IR data in Refs. 1 and 2.

Finally, the changes that take place in the position of the spectral peak of the $\mathrm{Si}-\mathrm{O}$ bond-stretching feature are consistent with the average $\mathrm{Si}-\mathrm{O}-\mathrm{Si}$ bond angle, $\beta$, increasing by about $5^{\circ}-10^{\circ}$, from about $145^{\circ}$ in $\mathrm{SiO}_{2}$ to more than $150^{\circ}$ in the $\mathrm{Si}-\mathrm{O}-\mathrm{F}$ alloys. The application of a nearest-neighbor, central force field analysis of the bond-stretching mode indicates that the frequency of this mode is directly proportional to the sine of one-half of the $\mathrm{Si}-\mathrm{O}-\mathrm{Si}$ bond angle; i.e., $\nu$ $=\nu_{0} \sin (\beta / 2)$, where $\nu_{0}$ is an empirically determined constant that is approximately equal to the square root of the ratio of a bond-stretching force constant and an oxygen atomic mass. ${ }^{9}$

\section{BONDING OF FLUORINE ATOMS IN Si-O-F}

The local atomic bonding of univalent atoms and groups in $\mathrm{SiO}_{2}$ has been previously addressed with emphasis on hydrogen $(\mathrm{H})$ atoms and hydroxyl $(\mathrm{OH})$ groups. In the F-doped $\mathrm{SiO}_{2}$ alloys, $\mathrm{F}$ atoms replace the $\mathrm{H}$ atoms or $\mathrm{OH}$ groups of hydrogenated $\mathrm{SiO}_{2}$. As noted above the $\mathrm{Si}-\mathrm{F}$ bondstretching vibration is at approximately the same frequency as that the $\mathrm{Si}-\mathrm{OH}$ vibration, $935 \mathrm{~cm}^{-1}$ as compared to $920 \mathrm{~cm}^{-1}$, consistent with the near equivalence of the masses of F (19 amu) and $\mathrm{OH}(17 \mathrm{amu})$. Figure 2 includes a schematic representation of the local atomic bonding of $\mathrm{Si}-\mathrm{F}$ groups in an $\mathrm{SiO}_{2}$ host. Based on the arguments presented above, there is no definitive IR spectroscopic evidence for $\mathrm{Si}-\mathrm{F}_{2}$ bonding in $\mathrm{Si}-\mathrm{O}-\mathrm{F}$ alloys with concentrations of $\mathrm{F}$ up to at least 12 at. \%. Therefore, the focus in what follows in based on changes in $\mathrm{Si}-\mathrm{O}-\mathrm{F}$ alloys with respect to $\mathrm{SiO}_{2}$ that are introduced by the incorporation of monofluoride or $\mathrm{Si}-\mathrm{F}$ bonding arrangements into the $\mathrm{SiO}_{2}$ network. The $\mathrm{Si}-\mathrm{F}$ bond is isoelectronic with the double-bonded $\mathrm{P}=\mathrm{O}$ group in $\mathrm{P}_{2} \mathrm{O}_{5}$ glasses, and the $\mathrm{Si}-\mathrm{F}$ bond-order is also greater than one due to back-donation from filled lone pair- $p$-orbitals of the $\mathrm{F}$ atom into empty anti-bonding states of Si with a $d^{3} s$ symmetry; i.e., the so-called $p \pi-d \pi$ bonding. ${ }^{10}$ The hydroscopic nature of the $\mathrm{Si}-\mathrm{O}-\mathrm{F}$ alloys derives from the high reactivity of the terminal $\mathrm{Si}-\mathrm{F}$ bond, paralleling the behavior of $\mathrm{P}=\mathrm{O}$ groups in $\mathrm{P}_{2} \mathrm{O}_{5}$ glasses and films.

The bonding model presented below has been constructed for an alloy regime in which each $\mathrm{Si}$ atom of a host $\mathrm{SiO}_{2}$ network is bonded to at most one F atom. As noted previously, there is no spectroscopic evidence for multiple attachment of $\mathrm{F}$ atoms as in $\mathrm{Si}-\mathrm{F}_{2}$ arrangements for the compositions of interest for low-dielectric constant device applications (up to $\sim 12$ at. $\% \mathrm{~F}$ ), so that the bonding model is clearly applicable for these alloy compositions. However to model alloy behavior, it is useful to consider higher $\mathrm{F}$ atom concentrations as well. If the monofluoride bonding is taken to the limit of one $\mathrm{F}$ atom/Si atom for the entire $\mathrm{SiO}_{2}$ network, then the resulting compound composition is given by $\mathrm{Si}_{2} \mathrm{O}_{3} \mathrm{~F}_{2}$ (this composition is an isoelectronic analog of the $\mathrm{P}_{2} \mathrm{O}_{5}$, which can be written as $\mathrm{P}_{2} \mathrm{O}_{3}\left(\mathrm{O}^{\prime}\right)_{2}$ in order to emphasize the two different $\mathrm{O}$ bonding environments, three bridging singly bonded oxygen atoms, $\mathrm{O}$, and one terminal doubly bonded oxygen atom designated as $\mathrm{O}^{\prime}$. Fluorinated oxides with lower concentrations of $\mathrm{F}$ can then be described as a homogeneous alloy mixture of $\mathrm{SiO}_{2}$ and $\mathrm{Si}_{2} \mathrm{O}_{3} \mathrm{~F}_{2}$, where a pseudo-binary alloy notation as in $\left(\mathrm{SiO}_{2}\right)_{x}\left(\mathrm{Si}_{2} \mathrm{O}_{3} \mathrm{~F}_{2}\right)_{1-x}$ is then appropriate. The resulting alloy compositions lie on the join-line between $\mathrm{SiO}_{2}$ and $\mathrm{Si}_{2} \mathrm{O}_{3} \mathrm{~F}_{2}$ in a ternary composition diagram. Note that once the at. \% of $\mathrm{F}$ is fixed, the ratio of $\mathrm{Si}$ to $\mathrm{O}$ is also determined. The number of $\mathrm{Si}-\mathrm{O}$ bonds/Si atom defined by $N_{\mathrm{Si}-\mathrm{O}}$ and is equal to $(6-2 x) /(2-x)$, and the number of $\mathrm{Si}-\mathrm{F}$ bonds/Si atom is defined by $N_{\mathrm{Si}-\mathrm{F}}$ is equal to $(2-2 x) /(2-x)$. Figure $3(\mathrm{a})$ contains $N_{\mathrm{Si}-\mathrm{O}}$ and $N_{\mathrm{Si}-\mathrm{F}}$ and Fig. 3(b) contains the atomic fractions, $[\mathrm{Si}]$, [O], and $[\mathrm{F}]$, all as functions of the alloy composition $x$. The same model, as shown in Fig. 4, can also be used to calculate the statistical probability for $\mathrm{F}$ atoms being

"isolated" in the context that they are on $\mathrm{Si}$ atoms that are separated by one or more $\mathrm{O}-\mathrm{Si}-\mathrm{O}$ groups,

at least paired; i.e., on $\mathrm{Si}$ atoms that are connected through a single $\mathrm{O}$ atom, and

(iii) more strongly clustered in the network structure.

The probability of $\mathrm{F}$ atoms being on $\mathrm{Si}$ atom sites that are isolated from other $\mathrm{F}$ atoms by at least one $\mathrm{O}-\mathrm{Si}-\mathrm{O}$ bonding group is given by $\rho(1-\rho)^{3}$, where $\rho$ is the fractional occupation of allowed $\mathrm{F}$ atom bonding sites. In a similar way the probability of a given $\mathrm{F}$ atom having at least one nearestneighbor $\mathrm{Si}$ site that is occupied by an $\mathrm{F}$ atom is given by $3 \rho^{2}(1-\rho)^{2}$. It is important to note that the $x$ axes in Figs. 3(a) and 3(b), and Fig. 4 are different. In Fig. 3, they reflect pseudo-binary alloy notation presented above, and in Fig. 4, the $x$ axis is related to the occupancy of available $\mathrm{F}$ bonding sites. 


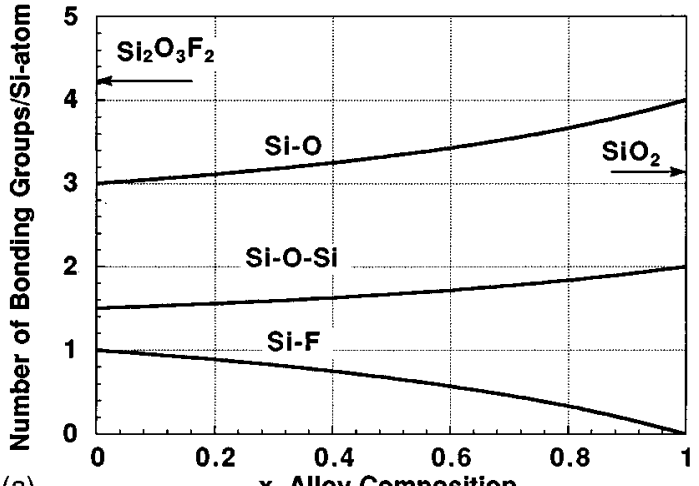

(a)

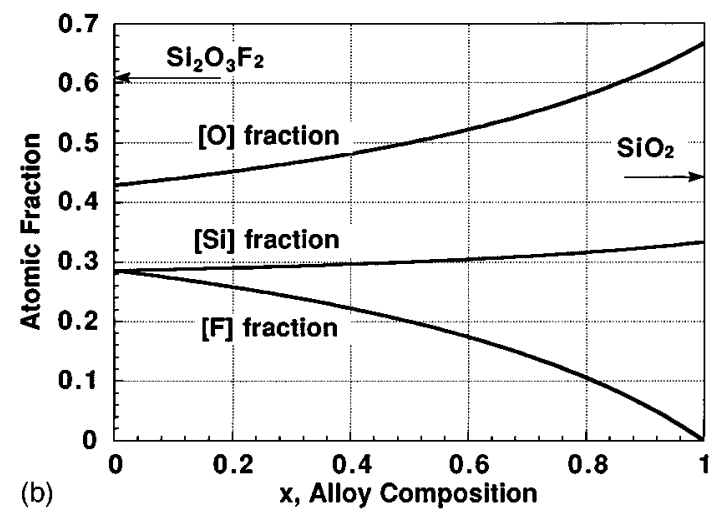

FIG. 3. (a) Concentrations of $N_{\mathrm{Si}-\mathrm{O}}$ and $N_{\mathrm{Si}-\mathrm{F}}$ bonds and $N_{\mathrm{Si}-\mathrm{O}-\mathrm{Si}}$ bonding groups, and (b) the atomic fractions, $[\mathrm{Si}],[\mathrm{O}]$, and $[\mathrm{F}]$; both are plotted as functions of $x$ in the pseudobinary alloy notation.

\section{DIPOLE OSCILLATOR CONTRIBUTIONS TO $\epsilon_{s}$}

In general $\epsilon_{s}$ can be expressed as a sum the contributions of dipole oscillators associated with electronic and vibrational transitions. A representation that is particularly useful is based on a sum of

(i) the optical frequency dielectric, $\epsilon_{0}$, which includes the integrated contributions from all of the electronic transitions, and

(ii) an additional sum over the contributions from specific types of IR-active vibrations.,

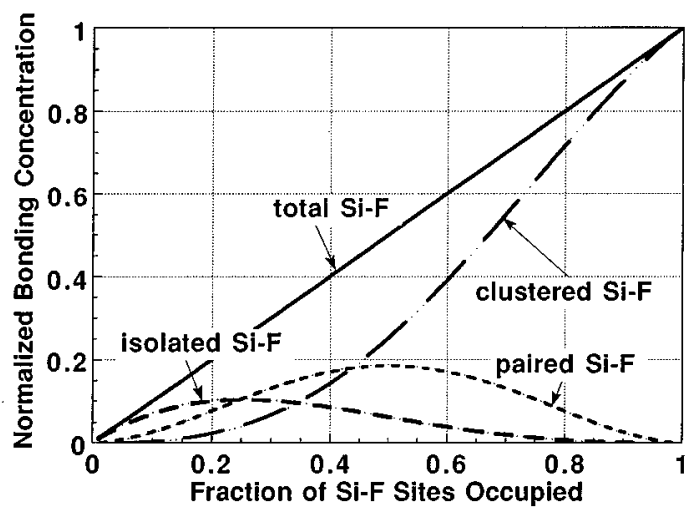

FIG. 4. Isolated and near-neighbor $\mathrm{Si}-\mathrm{F}$ concentrations as a function of $x$.
IR studies indicate significant reductions in absorption associated with $\mathrm{Si}-\mathrm{O}-\mathrm{Si}$ bond-stretching modes as $\mathrm{F}$ is added to the alloy ${ }^{1,2}$ as well as a weak IR response for the $\mathrm{Si}-\mathrm{F}$ bondstretching mode, so that the substitution of $\mathrm{F}$ for $\mathrm{O}$ by itself can lead to a significant reduction in the contribution of the vibrational modes to $\epsilon_{s}$. $\epsilon_{s}$ can be written as a three-band Penn model $^{5}$ as in Eq. (3):

$$
\epsilon_{s}=1+\left\{\frac{E p^{E}}{E g^{E}}\right\}^{2}+\left\{\frac{E p^{V \mathrm{SiO}}}{E g^{V \mathrm{SiO}}}\right\}^{2}+\left\{\frac{E p^{V \mathrm{SiF}}}{E g^{V \mathrm{SiF}}}\right\}^{2},
$$

where

(i) $E p^{E}$ is an effective electronic plasma frequency,

(ii) $E g^{E}$ is an effective optical band-gap, and

(iii) $E p^{V X}$ and $E g^{V X}$ are vibrational frequency analogs of $E p^{E}$ and $E g^{E}$, reflecting the separate contributions from $X=\mathrm{Si}-\mathrm{O}$ and $\mathrm{Si}-\mathrm{F}$ vibrations.

The vibrational plasma frequencies, $E p^{V X}$, represent integrated oscillator strengths that are proportional to the bandwidths and the squares of infrared effective charges, and the vibrational band gaps, $E g^{V X}$, are effective vibrational frequencies that are approximately equal to the positions of the respective bond-stretching and bending features in the absorption or $\epsilon_{2}$ spectra (see Fig. 1 and Refs. 1 and 2). The first two terms in Eq. (3) are defined as the optical frequency dielectric constant, $\epsilon_{0}$ :

$$
\epsilon_{0}=1+\left\{\frac{E p^{E}}{E g^{E}}\right\}^{2}
$$

Note that $\epsilon_{0}$ is the square of the index of refraction, $n$, which is approximately constant in the transparent region between the band edge and the vibrational absorption bands. Specific relationships between the vibrational and plasma terms in Eq. (4) and vibrational frequencies and IR effective charges can be obtained from

the macroscopic equations that relate transverse optical (TO) and longitudinal optical (LO) frequencies to IR oscillator strengths, and

(ii) the Lydanne, Sachs, Teller relationship as presented for example in Ref. 5.

Note that for disordered materials such as $\mathrm{SiO}_{2}$ and $\mathrm{Si}-\mathrm{O}-\mathrm{F}$ alloys, the effective TO frequencies are defined by the spectral peaks in the imaginary part of the dielectric function, $\epsilon_{2}$, and the effective LO frequencies are defined by the spectral peaks in the energy loss function, $\operatorname{Im}(-1 / \epsilon)$. The squares of the vibrational plasma frequencies, $E p^{V X}$, are proportional to the square of the respective infrared effective charges, and the film density, and the squares of the vibrational band gap terms, $E g^{V X}$, are proportional to the squares of the respective transverse vibrational frequencies. These frequencies are integrated averages, and in the spirit of the Penn model, their exact positions are noted necessarily exactly the same as the spectral peaks in the absorption or $\epsilon_{2}$ spectra. ${ }^{5}$ However, for purposes of this article, it is important 
to understand that increases in the position of the spectral absorption peaks will also produce increases in the corresponding $E g^{V X}$ terms.

\section{REDUCTIONS IN $\epsilon_{s}$ AS A FUNCTION OF FORMAL BOND IONICITY}

To illustrate how $\epsilon_{s}$ scales with an empirically defined bond ionicity, the contributions from electronic and vibrational excitations are presented for a series of Si-based materials in which formal bond ionicity increases: crystalline $\mathrm{Si}$, crystalline $\mathrm{Si}-\mathrm{C}$, amorphous $\mathrm{SiO}_{2}$, and $\mathrm{Si}_{3} \mathrm{~N}_{4}$, and amorphous $\mathrm{Si}-\mathrm{O}-\mathrm{F}$ alloys. An empirically defined ionicity for each of these materials can be characterized by the partial charge on the Si atom that is calculated using the electronegativities of the constituent atoms. This partial charge represents a 'formal' charge transfer from the $\mathrm{Si}$ atom in forming chemical bonds to the other compound or alloy constituents; i.e., the larger the electronegativity difference, the larger the partial charge on the $\mathrm{Si}$ atom. It is important to note that this partial charge is not equal to an infrared effective charge which, as shown below, generally has both static and dynamic contributions. In addition, and more importantly, following the original definition of electronegativity as put forth by Pauling, the larger the partial charge on the $\mathrm{Si}$ atom, the more ionic the bonding in the context of heteropolar contributions to the total bond energy. ${ }^{10}$

Using the Sanderson method of Ref. 11, an effective electronegativity $\left(X_{A}^{\prime}\right)$ can be calculated for each of the materials in a compound $\left(A_{n} B_{m}\right)$ according to the relationship presented below in Eq. (5):

$$
X_{A}^{\prime}=X_{B}^{\prime}=\left[\left(X_{A}\right)^{n}\left(X_{B}\right)^{m}\right]^{1 /(n+m)},
$$

where $A$ is taken to be $\mathrm{Si}$, and $B$ is the other atomic constituent; e.g., C, N, O or F. Using the values of $X_{\mathrm{Si}}^{\prime}$ for $\mathrm{Si}$ from Eq. (5), the Si-atom partial charge, $e_{\mathrm{Si}}$, is then calculated from:

$$
e_{\mathrm{Si}}=\frac{X_{\mathrm{Si}}^{\prime}-X_{\mathrm{Si}}}{2.08\left(X_{\mathrm{Si}}\right)^{0.5}},
$$

where $X_{\mathrm{Si}}$ is the electronegativity of a $\mathrm{Si}$ atom in a purely covalent bond, as in crystalline Si. ${ }^{11}$ Equation (6) is an empirical relationship that is based on defining effective charges for $\mathrm{Na}$ and $\mathrm{F}$ in $\mathrm{NaF}$ that are consistent with the fractional ionicity of that material (see details in Ref. 8). The values of $e_{\mathrm{Si}}$ for materials other than crystalline $\mathrm{Si}$ considered in Figs. 5(a) and 5(b) are positive indicating electrons are withdrawn from the $\mathrm{Si}$ atom when it bonds to species such as $\mathrm{C}, \mathrm{N}, \mathrm{O}$ and $\mathrm{F}$ which have values of electronegativity that are greater than that of $\mathrm{Si}$; i.e., from Eq. (5) $X_{\mathrm{Si}}^{\prime}$ is larger than $X_{\mathrm{si}}$, so that $e_{\mathrm{Si}}$ is positive. Figure 5(a) contains plots of experimentally determined values of $\epsilon_{0}$ and $\epsilon_{S}$ as functions of the $\mathrm{Si}$ atom partial charge; $\epsilon_{0}=n^{2}$, where $n$ is the index of refraction. The difference between $\epsilon_{s}$ and $\epsilon_{0}$ is due primarily to the contributions from the vibrational modes and is shown in Fig. 5(b). In some molecular systems there are also contributions to $\epsilon_{s}$ from very low frequency dipolar effects; however, there is no evidence that these contributions are signifi-
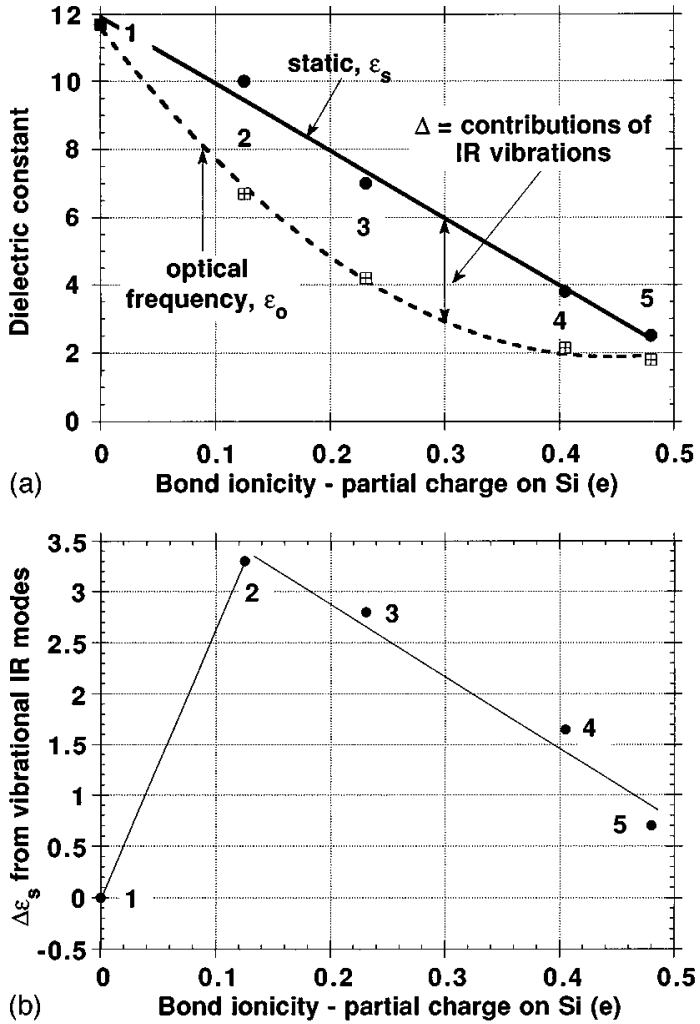

FIG. 5. Plots of (a) $\epsilon_{0}$ and $\epsilon_{s}$ and (b) the contribution to $\epsilon_{s}$ from the vibrational modes, both as functions of the $\mathrm{Si}$ atom partial charge for: 1 crystalline-Si, $2 \beta$-SiC, 3 noncrystalline $\mathrm{Si}_{3} \mathrm{~N}_{4}, 4$ noncrystalline $\mathrm{SiO}_{2}$, and 5 extrapolated approximate values for $\mathrm{Si}_{2} \mathrm{O}_{3} \mathrm{~F}_{2}$. The values of $\epsilon_{0}\left(=n^{2}\right)$ and $\epsilon_{s}$ are experimental.

cant in inorganic materials such as $\mathrm{SiO}_{2}$ and $\mathrm{Si}_{3} \mathrm{~N}_{4}$. Returning to the IR active vibrational modes, as the partial charge of the $\mathrm{Si}$ atom increases, the contribution of the vibrational modes to $\epsilon_{s}$ in Fig. 5(b) decreases. This observation is counter-intuitive in as much as it sometimes assumed that the strength of IR active modes increases as the bond ionicity increases. Many studies have shown that this is not the case; e.g., the IR active modes in homopolar trigonal Se and Te crystals make contributions to the static dielectric constant well in excess of those of ionic crystal such as $\mathrm{NaCl}^{12}$ This derives from the fact that the IR effective charges, that characterize the IR strengths have both static contributions, that can sometimes correlate with bond ionicity as defined above, as well as dynamic contributions that come from charge redistribution during the course of the vibrational motion (see discussion below). For Se and Te the effective charges are entirely dynamic and are significantly larger than the effective charges in ionic materials such as $\mathrm{NaCl}$ and other rocksalt structured crystals. ${ }^{12}$

As noted above the contribution of a vibrational mode to $\epsilon_{s}$ depends on the ratio of a plasma frequency to a vibrational band gap or average TO frequency. Two factors contribute to the trend in Fig. 5(b)

(i) decreases in the IR effective charge with increasing bond ionicity, and 


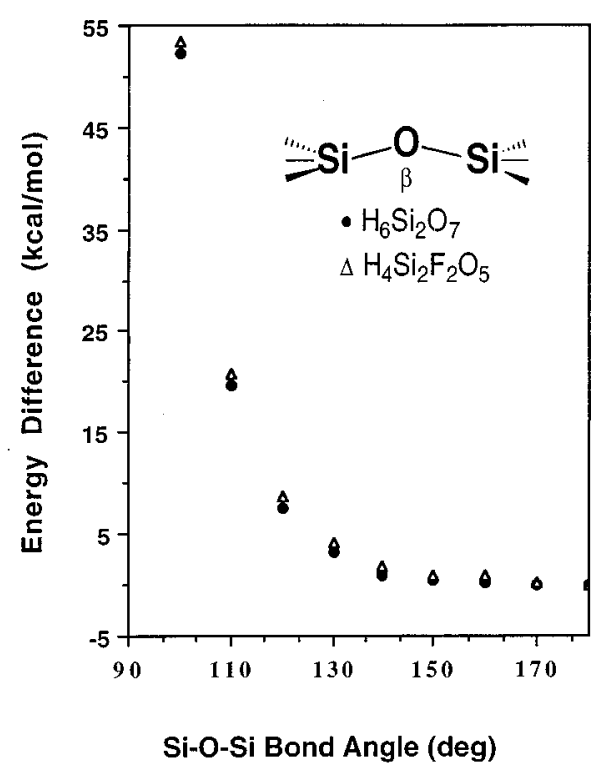

FIG. 6. Excess energy with respect to optimum energy as a function of $\mathrm{Si}-\mathrm{O}-\mathrm{Si}$ bond angle, $\beta$. This diagram contains a schematic representation of the two molecular structures used in the ab initio calculations.

(ii) increases in the effective TO frequency with increasing ionicity.

The decreases in the IR effective charges reduce the vibrational effective plasma frequencies and the increases in the effective TO frequencies increase the effective vibrational band gaps. Referring to Eq. (3), these changes both contribute to decreases in $\epsilon_{s}$. As shown in Sec. VI, there are two factors related to effective charges contribute to the decreased IR contributions

(i) the IR effective charge of a $\mathrm{Si}-\mathrm{F}$ vibration is smaller than that of the $\mathrm{Si}-\mathrm{O}-\mathrm{Si}$ vibration that it replaces, and

(ii) the incorporation of a $\mathrm{Si}-\mathrm{F}$ bond to a $\mathrm{Si}$ atom that is back-bonded to three $\mathrm{O}$ atoms can reduce the IR effective charges of the back-bonded $\mathrm{Si}-\mathrm{O}-\mathrm{Si}$ groups.

A third factor derives from a significant increase in the frequency of the bond-stretching $\mathrm{Si}-\mathrm{O}-\mathrm{Si}$ upon alloying with F. It is important to recognize that in applying the extended Penn model of Eq. (3), that the plasma frequency terms represent integrals over vibrational bands, e.g., the bending, stretching, and rocking modes of $\mathrm{SiO}_{2}$, and as such are not simply proportional to the peak values of the respective absorption constants.

\section{RESULTS OF AB INITIO CALCULATIONS}

The details of the $a b$ initio calculations for the effects identified immediately above in (i) and (ii) will be presented elsewhere. ${ }^{13}$ At this time, we will simply indicate the important results for

(i) the energy difference between the minimum value for a $180^{\circ} \mathrm{Si}-\mathrm{O}-\mathrm{Si}$ bond angle $(\beta)$ and other bond angles down to the $100^{\circ}$ in Fig. 6, and (ii) the changes in the IR effective charges for the $\mathrm{Si}$ $\mathrm{O}-\mathrm{Si}$ bond-stretching, bending, and rocking vibrations in Fig. 7 that are induced by $\mathrm{F}$ atom induction effects in Fig. 7.

Figure 6 displays the energy difference between the minimum value for a $180^{\circ} \mathrm{Si}-\mathrm{O}-\mathrm{Si}$ bond angle $(\beta)$ and other bond angles down to $100^{\circ}$. A schematic representation of the local clusters used in this calculation is included. There is no significant difference in this energy difference for $\mathrm{Si}-\mathrm{O}-\mathrm{Si}$ bond angles between about $130^{\circ}$ and $180^{\circ}$, consistent with x-ray diffraction studies of glassy $\mathrm{SiO}_{2}$ which indicate an average bond angle of about $150^{\circ}$, and a bond angle spread of about $50^{\circ} .{ }^{14}$ Most importantly for purposes of this study this difference is in the variation of this energy difference function for $\mathrm{H}$ terminated and $\mathrm{F}$ terminated clusters.

The IR effective charges presented in Figs. 7(a), 7(b) and 7 (c) are computed numerically by calculating changes in the dipole moments $(M)$ corresponding to displacements $(u)$ of the $\mathrm{O}$ atom and $\mathrm{F}$ atoms that are appropriate to their respective bonding stretching vibrations; i.e., the $\mathrm{O}$ atom is displaced in direction parallel a line joining its two $\mathrm{Si}$ atom neighbors, and the $\mathrm{F}$ atom is displaced along the $\mathrm{Si}-\mathrm{F}$ bond. The IR effective charge $\left(e^{*}\right)$ is associated with a redistribution of the electrons that results from these displacements, and is given by the following expression:

$$
e^{*}=\delta M / \delta u,
$$

where the derivative is evaluated in the limit of small displacements of $u$; i.e., one calculates the moment, $M$, as a function of the displacement coordinate, $u$, and then determines the derivative in the limit of small displacements. This calculation is not in any way based on the definition of partial charge as determined from the atomic electronegativities, nor is it expected that the IR effective charge should be equal to the IR effective charge. The IR effective charge is a derivative property of the local atomic structure, whereas the partial charge is a static property that is directly related to ionic contributions to the total bond energy. The contribution of a vibrational mode to $\epsilon_{s}$ is proportional to the square of this effective charge and these are the quantities presented in Fig. 7. Note that the bond angles of interest are from about $120^{\circ}-180^{\circ}$ degrees.

Consider first the bond-stretching modes: the square of the IR effective charge for this mode

is a strong function of the $\mathrm{Si}-\mathrm{O}-\mathrm{Si}$ bond angle, increasing by more than a factor of 2 as the bond angle is increased from about $120^{\circ}$ to $180^{\circ}$ and

(ii) is reduced by about the same amount for all angles by $\mathrm{F}$ addition.

The effective charge of the bond-bending mode shows a complementary behavior, decreasing by about $30 \%$ as the bond angle is increased. As in the case of the bond-stretching mode, the addition of $F$ reduces the effective charge for all $\mathrm{Si}-\mathrm{O}-\mathrm{Si}$ bond angles considered; however the decrease be- 


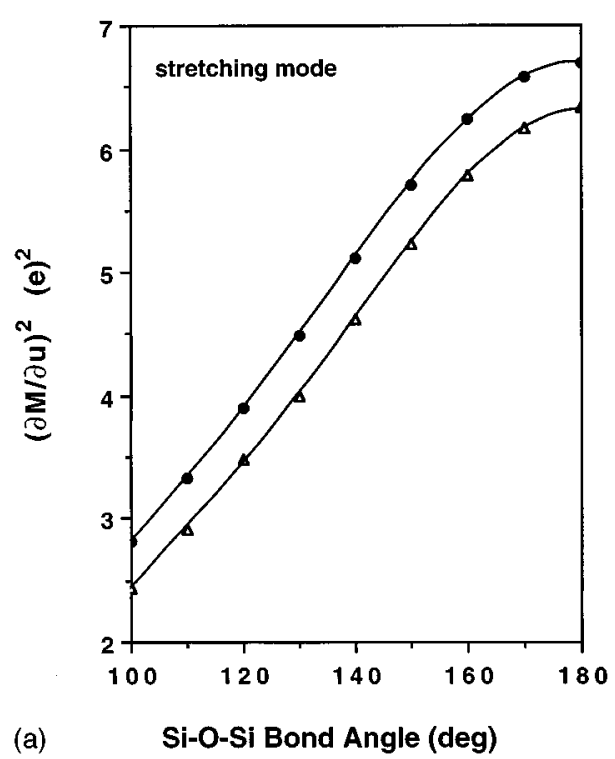

(a) Si-O-Si Bond Angle (deg)

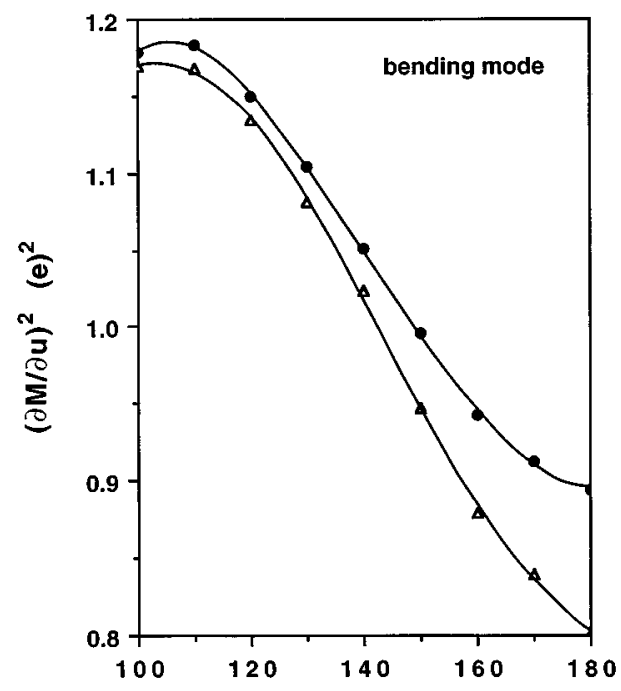

(b) Si-O-Si Bond Angle (deg)

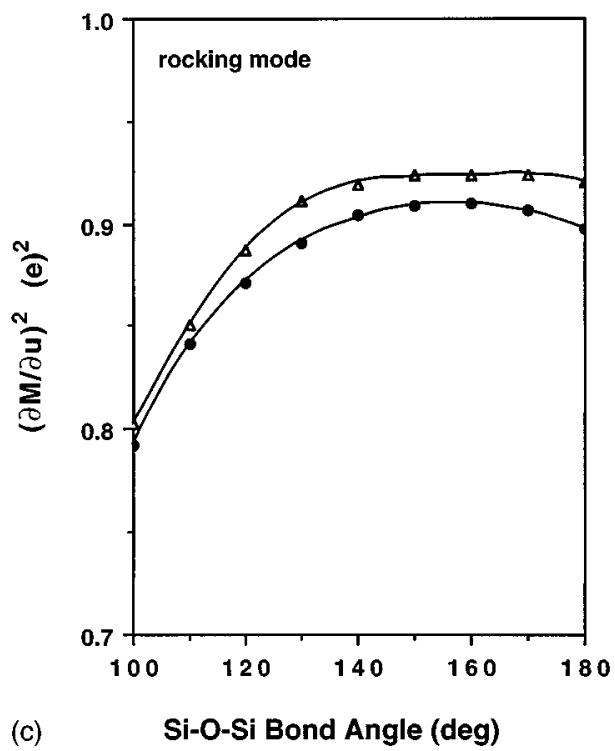

FIG. 7. Squares of the IR effective charges for (a) bond-stretching, (b) bondbending, and (c) bond-rocking modes as a function of the $\mathrm{Si}-\mathrm{O}-\mathrm{Si}$ bond angle. The diagrams contain comparisons. comes much greater as the $\mathrm{Si}-\mathrm{O}-\mathrm{Si}$ angle increases. Finally, the effective charge for the bond-rocking mode shows a qualitatively different behavior

(i) there is a small increase in the effective charge for the fluorinated case, and

(ii) the variation of the effective charge with $\mathrm{Si}-\mathrm{O}-\mathrm{Si}$ bond angle is significantly smaller than the for the bond-bending and bond-stretching modes, particularly for bond angles between about $120^{\circ}$ and $180^{\circ}$.

With regard to the relative magnitudes of the effective charges, the bond-bending and bond rocking modes are have similar effective charges, $\sim 1 e$ in magnitude, whereas the effective charge for the bond-stretching mode is significantly greater, $\sim 2-2.5 e$ for $\mathrm{Si}-\mathrm{O}-\mathrm{Si}$ bond angles in the vicinity of $150^{\circ}$. The relative magnitudes of the squares of the calculated effective charges for the three vibrational modes of $\mathrm{SiO}_{2}$ are in excellent agreement with the integrated strengths of the respective IR bands.

From the experimental data presented in Refs. 1 and 2 and the results in Fig. 1, it is obvious that the reduction in the $\mathrm{Si}-\mathrm{O}-\mathrm{Si}$ vibrational mode contributions to $\epsilon_{s}$ from the substitution of one $\mathrm{F}$ atom for one $\mathrm{O}$ atom is insufficient to account for the relatively large decreases in $\epsilon_{s}$. For example, in a 12 at. $\% \mathrm{~F}$ alloy which exceeds the concentration discussed above, about $10 \%$ of the $\mathrm{Si}-\mathrm{O}-\mathrm{Si}$ groups are removed, and the maximum decrease in $\epsilon_{s}$ from removal of these groups is about $10 \%$ of $\delta \epsilon=0.1\left(\epsilon_{s}-\epsilon_{0}\right)$ $\approx 0.10(4.1-2.1)$ or $\sim 0.2$, whereas the reported decrease in $\epsilon_{s}$ is approximately 0.8 . This zeroth order calculation actually overestimates the effect for the bond-stretching modes, where the modes most effected are those with the smallest bond angles and the smallest IR moments. The reverse is true for the bond-bending modes, and the anticipated fractional decrease in this mode at $\sim 800 \mathrm{~cm}^{-1}$ is readily evident in the IR spectra (see Refs. 1 and 2). Since the square of the moment of bond-stretching modes of the $\mathrm{Si}-\mathrm{O}-\mathrm{Si}$ groups bonded to a $\mathrm{Si}-\mathrm{F}$ are reduced by the addition of that $\mathrm{F}$ neighbor, this adds significantly to the decrease in $e_{s}$. Since for $40 \%$ occupancy of the available $\mathrm{Si}$ atom bonding sites, each $\mathrm{Si}-\mathrm{O}-\mathrm{Si}$ groups is in bonding contact with at least one $\mathrm{F}$ atom, this mechanism contributes to an additional decrease of about 0.05 , that is in part offset by the shift in the centroid of the bond-stretching modes to higher wave number. Combined with the decreased contributions from the $\mathrm{Si}-\mathrm{O}-\mathrm{Si}$ bending modes which contribute to an additional decrease in $\epsilon_{s}$ of $\sim 0.1$, this approach goes far in accounting for the behavior of $\epsilon_{s}$ with increasing $\mathrm{F}$ content. However, to be more quantitative, two additional factors must be taken into account. One additional factor is the increase in the vibrational frequency of the $\mathrm{Si}-\mathrm{O}-\mathrm{Si}$ bond-stretching mode [see Eq. (2)]. For example, increasing the average frequency of the $\mathrm{Si}-\mathrm{O}-\mathrm{Si}$ bond-stretching vibration from 1060 to $1090 \mathrm{~cm}^{-1}$ will decrease the fractional contribution of these modes to $\epsilon_{s}$ by $\sim 6 \%$, or about 0.15 . Since these modes make the largest contribution to $\epsilon_{s}$, this change is indeed significant. Finally, as noted above, the reduction in alloy 
density, $\sim 5 \%$ for an alloy with $10-12$ at. $\% \mathrm{~F}$ also contributes a factor of $\sim 0.2 .{ }^{4}$ Combining these terms then explains a total decrease in $\epsilon_{s}$ of about 0.7 , as compared to the experimental value of 0.8 . Combining these terms then explains the significant decreases in $\epsilon_{s}$. The quantitative agreement validates the accuracy of the theory and the use of relatively small molecular clusters to obtain properties of vibrational modes in continuous random networks.

The calculation presented above identifies the answer to the apparent paradox between increases in the $\mathrm{Si}$ partial charge and decreases in the contribution of vibrational modes to $\epsilon_{s}$. We had shown in Figs. 5(a) and 5(b) that the contribution of vibrational modes to $\epsilon_{s}$ decreased with increasing partial charge on the $\mathrm{Si}$ atom. It must be noted that this partial charge is merely an empirical way of characterizing chemical bonding effects associated with electronegativity changes induced by near-neighbor atoms. The validity of this approach is borne out by the scaling of vibrational frequencies of $\mathrm{Si}-\mathrm{H}$ (Ref. 5) and $\mathrm{P}=\mathrm{O}$ (Ref. 10) vibrations, and deep level core shifts as monitored by $\mathrm{x}$-ray photoelectron spectroscopy. ${ }^{10}$ On the other hand, this partial charge is not necessarily equal to, or even proportional to the IR effective charge. The large IR effective charges, $\sim 2.5 e$ for bond angles of about $150^{\circ}$, are significantly larger than $e_{\mathrm{Si}}$. From the $a b$ initio calculations, it is evident that the large IR charge for the asymmetric bond-stretching vibration is due to a dynamic effect rather than static charge transfer. On the other hand, the smaller IR effective charge for the bondrocking mode is due mostly to static charge transfer. The $a b$ initio calculations also provide direct evidence for the back donation of electrons from the $\mathrm{F}$ atom to the $\mathrm{Si}$ atom, confirming the empirical chemistry arguments for a $p \pi-d \pi$ component of the $\mathrm{Si}-\mathrm{F}$ bonding. This is the effect which serves to reduce the dynamic effective charge of the $\mathrm{Si}-$ $\mathrm{O}-\mathrm{Si}$ groups when $\mathrm{F}$ nearest neighbors are present.

\section{REACTION PATHWAYS TO FLUORINE ATOM INCORPORATION}

There are two possible reaction pathways to $\mathrm{F}$ atom incorporation in the $\mathrm{SiO}_{2}$ materials:

development of an $\mathrm{SiO}_{2}$ network structure with subsequent attack by $\mathrm{F}$, or

(ii) the development of the $\mathrm{Si}-\mathrm{O}-\mathrm{F}$ network directly.

The major effects of the added $\mathrm{F}$ atoms on the $\mathrm{SiO}_{2}$ network as exemplified by the IR is the shift of the $\mathrm{Si}-\mathrm{O}-\mathrm{Si}$ bondstretching frequency to higher wave numbers and the accompanying reduction of the spectral width of that feature. Since the spectral peak absorbance of the bond-stretching mode in the fluorinated films is higher than the absorbance at the same wave number in the film without any F (see Fig. 1 and IR data in Ref. 1), this means that the second mechanism applies; that is the IR absorption in the fluorinated film is not simply related to removing some of the oscillators that contribute to that band. The situation is different in $\mathrm{SiO}_{2}$ films that have been attacked by water vapor. ${ }^{8}$ In particular, the first mechanism applies to the attack by water of low tem- perature, low density $\mathrm{SiO}_{2}$ films prepared by plasma deposition. ${ }^{6}$ This post deposition process is characterized by its reversible character as well.

\section{DISCUSSION}

The main points of this article are

(i) for relatively small additions of $\mathrm{F}$ to $\mathrm{SiO}_{2}$, up to 12 at. $\%$, there are relatively large changes in $\epsilon_{s}$,

(ii) alloy compositions with small additions of $\mathrm{F}$ can be described in terms of a pseudobinary notation, $\left(\mathrm{SiO}_{2}\right)_{x}\left(\mathrm{Si}_{2} \mathrm{O}_{3} \mathrm{~F}_{2}\right)_{1-x}$, from which alloy atom and bonding configurations can be computed;

(iii) the primary factor contributing to decreases in $\epsilon_{s}$ in these alloys is a decrease in the contributions from the vibrational modes;

(iv) the replacement of $\mathrm{O}$ with $\mathrm{F}$ has two effects on the IR effective charges which determine the absorption strengths:

(a) $\mathrm{Si}-\mathrm{F}$ vibrations have lower $e^{*}$ 's for the dominant bond-stretching modes than $\mathrm{Si}-\mathrm{O}$ vibrations, and

(b) the increased partial charge on the $\mathrm{Si}$ reduces the $e^{*}$ 's of the both the bond-stretching and bondbending vibrations $\mathrm{Si}-\mathrm{O}$ vibrations;

(i) the hydroscopic nature of the $\mathrm{Si}-\mathrm{O}-\mathrm{F}$ alloys is related to $\mathrm{Si}-\mathrm{F}$ bonding, predominantly near-neighbor $\mathrm{Si}-\mathrm{F}$ groups; and

(ii) there are no other inorganic dielectrics based on alloy formation with $\mathrm{SiO}_{2}$ that can yield lower values of $\epsilon_{s}$, so that the alloy approach to the so-called low- $k$ dielectrics is limited to reductions of $\epsilon_{s}$ to values between 3.2 and 3.4 .

\section{ACKNOWLEDGMENTS}

This work is supported by ONR, National Science Foundation, SRC, and Intel Corporation.

${ }^{1}$ S. W. Lim, Y. Shimogaki, Y. Nakano, K, Tada, and H. Komiyama, Extended Abstracts of the 1995 International Conference on Solid State Device and Materials (Business Center for Academic Societies Japan, Tokyo, 1995), p. 153.

${ }^{2}$ S. W. Lim, Y. Shimogaki, Y. Nakano, K. Tada, and H. Komiyama, Jpn. J. Appl. Phys. 135, 1468 (1996).

${ }^{3}$ G. Lucovsky, AIP. Conf. Proc. 75, 100 (1981), and references therein.

${ }^{4}$ D. T. Hodul, MRS Symp. Proc. (in press).

${ }^{5}$ G. Burns, Solid State Physics (Academic, New York, 1985).

${ }^{6}$ N. Hayasaka, H. Miyajima, Y. Nakasaki, and R. Katsumaa, Extended Abstracts of the 1995 International Conference on Solid State Device and Materials (Business Center for Academic Societies Japan, Tokyo, 1995), p. 157.

${ }^{7}$ G. Lucovsky, in Fundamentals Physics of Amorphous Semiconductors, edited by F. Yonezawa (Springer, Berlin, 1980), p. 87.

${ }^{8}$ J. A. Theil, D. V. Tsu, S. S. Kim, and G. Lucovsky, J. Vac. Sci. Technol. A 8, 1374 (1990).

${ }^{9}$ G. Hertzberg, Infra-red and Raman Spectra of Polyatomic Molecules (Van Nostrand, New York, 1945).

${ }^{10}$ J. E. Huheey, Inorganic Chemistry (Harper and Row, New York, 1978).

${ }^{11}$ R. T. Sanderson, Chemical Bonds and Bond Energy (Academic, New York, 1971).

${ }^{12}$ E. Burstein, M. H. Brodsky, and G. Lucovsky, Int. J. Quantum Chem. 1s, 759 (1967).

${ }^{13}$ H. Yang, G. Lucovsky, and J. L. Whitten (unpublished).

${ }^{14}$ R. L. Mozzi and B. E. Warren, J. Appl. Crystallogr. 2, 164 (1969). 